\title{
Past, present, and future of the pathophysiological model of the basal ganglia
}

\author{
José A. Obeso ${ }^{1,2^{*}}$ and José L. Lanciego $0^{1,2^{*}}$ \\ ${ }^{1}$ Neurosciences Division, Center for Applied Medical Research, University of Navarra, Pamplona, Spain \\ ${ }^{2}$ Centro de Investigación Biomédica en Red sobre Enfermedades Neurodegenerativas, Pamplona, Spain
}

\section{Edited by:}

Enrico Mugnaini, Northwestern

University, USA

\section{Reviewed by:}

Stefano Taverna, Italian Institute of Technology, Italy

Espen Dietrichs, University of Oslo, Norway

\section{*Correspondence}

José A. Obeso and José L. Lanciego Neurosciences Division, Center for

Applied Medical Research, University

of Navarra, Pio XII Avenue 55, 31008

Pamplona, Spain.

e-mail: jobeso@unav.es;

jlanciego@unav.es
The current model of basal ganglia (BG) was introduced two decades ago and has settled most of our current understanding of BG function and dysfunction. Extensive research efforts have been carried out in recent years leading to further refinement and understanding of the normal and diseased BG. Several questions, however, are yet to be resolved. This short review provides a synopsis of the evolution of thought regarding the pathophysiological model of the BG and summarizes the main recent findings and additions to this field of research. We have also tried to identify major challenges that need to be addressed and resolved in the near future. Detailed accounts and state-of-the-art developments concerning research on the BG are provided in the articles that make up this Special Issue.

\section{Keywords: Parkinson's disease, dopamine, subthalamic nucleus, caudate putamen, substantia nigra}

\section{INTRODUCTION}

The basal ganglia (BG) have been traditionally linked to the control of movement. This mindset was mainly derived from early clinico-pathological observations of BG lesions associated with movement disorders and, subsequently, by the profound impact that striatal dopamine (DA) depletion caused both in animals and in patients with Parkinson's disease (PD).

The first coherent model of the BG was developed in the mid1980s (Penney and Young, 1986; Albin et al., 1989; DeLong, 1990) whereupon the BG were shown to act by integrating and processing information through a series of connections with different brain regions. This process involves the conduction of information from the cerebral cortex and thalamus to the striatum, then to the globus pallidus pars interna ( $\mathrm{GPi}$ ) and substantia nigra pars reticulara $(\mathrm{SNr})$, to provide feed-back via the ventral thalamus to the cerebral cortex and the superior colliculus. This work was preceded by fundamental anatomical and physiological studies in monkeys describing the existence of segregated cortico-BGthalamo-cortical circuits mediating different functions, as determined by the cortical area of origin. These circuits comprised the skeletomotor ("motor"), oculomotor, associative, and limbic circuits, whose general anatomical organization was similar, but whose cortical and sub-cortical component regions were distinct (Alexander et al., 1986). In simple terms, the BG were seen as a "go through" station that receives, processes and conveys information via closed and parallel loops.

\section{THE PAST}

The model was based on two fundamental concepts which led to the formulation of a hypothesis of how the BG function.

The first concept was settled on the preponderance of anatomi$\mathrm{cal}$ and neurochemical data available at the time, and based mainly on the fact that different populations of striatal medium spiny neurons (MSNs) project to the output of the BG (GPi and $\mathrm{SNr}$ ) via "direct" and "indirect" pathways (Alexander et al., 1986; Penney and Young, 1986; Albin et al., 1989; DeLong, 1990). The "direct" pathway is a monosynaptic projection arising from MSNs that express D1 receptors, substance $\mathrm{P}$ and dynorphin, exerting a phasic and robust inhibitory effect on GPi/SNr neurons. The "indirect" pathway stems from MSNs expressing D2 receptors and enkephalin that project to the $\mathrm{GPi} / \mathrm{SNr}$ through a polysynaptic ("indirect") pathway that involves relays in the external globus pallidus (GPe) and the subthalamic nucleus (STN).

The second concept was founded on physiological data. $\mathrm{GPi} / \mathrm{SNr}$ neurons fire in a mostly tonic manner, keeping targeted structures in the thalamus and brainstem under tonic inhibitory control. Brief pauses in neuronal activity in the output of the BG facilitate movement (for example, saccadic eye movement) whereas neuronal discharges inhibit or halt movements (Chevalier and Deniau, 1990). Dopamine, from the nigro-striatal projection, was postulated to have a differential effect on D1/D2-expressing MSNs, facilitating and inhibiting respectively the "direct" and "indirect" pathways (Gerfen et al., 1990). In this so-called "rate model" it was thus postulated that in the normal state, activation of the "direct" pathway facilitates movement whereas activation of the "indirect" pathway inhibits or stops movement. Dopaminergic depletion, the key feature of the parkinsonian state, reduces the facilitation of MSNs belonging to the "direct" projection and enhances the activation of "indirect" pathway neurons, leading to increased activity of the STN which in turns overactivates inhibitory output neurons in the GPi/SNr. In this way, the combined reduction of GABA inhibition from the "direct" striatal output neurons and increased glutamatergic driving from the dis-inhibited STN led to increased activity in the GPi/SNr, 
effectively reducing the likelihood of phasic inhibitory activity in these output neurons, and thereby impeding movement initiation and execution. Seminal studies in the monkey by Crossman and co-workers led to a similar understanding for the dyskinetic state (i.e., hemichorea-ballism and levodopa-induced dyskinesias), essentially considered to result from decreased GPi output and therefore representing a functional mirror of the parkinsonian state (Crossman, 1990).

The "rate" model of BG dysfunction in parkinsonism, and particularly the proposed role of increased activity in the STN and GPi was strongly supported by studies showing that subthalamotomy in MPTP-treated monkeys greatly ameliorated parkinsonian motor features and normalized BG output abnormalities (Aziz et al., 1991; Wichmann et al., 1994; Guridi et al., 1996). These findings reignited interest in the use of ablative stereotactic surgery in PD patients with levodopa-induced motor complications. Thus, in the 1990s unilateral pallidotomy firstly, and subthalamotomy subsequently, were shown to be highly effective in the treatment of patients with PD. This in turn led to the application of highfrequency stimulation (“deep brain stimulation," DBS), which was initially employed in the thalamus to treat tremor. DBS has several advantages over classic ablative surgery; for instance it can be performed bilaterally without major side effects and is potentially reversible.

The surgical experience also provided ample sources of published information and analysis, and led to two well-recognized paradoxes. The first paradox rests in the fact that lesion of the postero-lateral GPi, and thus interrupting BG output to the motor thalamus, does not cause dyskinesias (Obeso et al., 1997). Indeed, pallidotomy eliminates dyskinesias, which is exactly the opposite effect of the model prediction. The second paradox concerns the observation that lesion of the motor thalamus does not aggravate PD (Marsden and Obeso, 1994); furthermore, elimination of BG output nuclei by lesion of the GPi and STN is not associated with any major clinical deficit. Recently, some light has been shed on these conflicting observations. On the one hand, the "firing rate" based model has been expanded to consider that the degree of neuronal synchronization and firing patterns are also important determinants of the motor state (Brown, 2003). Normally, BG neurons discharge asynchronously, but in the parkinsonian state hypersynchrony of neuronal activity in the BG output nuclei and cortex is present. Recording local field potentials (LFPs) through DBS macro-electrodes implanted in the STN has shown enhanced beta-frequency (mean $\sim 18 \mathrm{~Hz}$ ) oscillations in the "off" motor state. Such beta activity is reduced in the "on" medication state while gamma-band ( $>60 \mathrm{~Hz}$ ) activity is increased (Brown et al., 2001). Subsequent studies have documented that similar oscillations also occur in the GPi and cortex in PD patients (Brown et al., 2001). Moreover, abnormal oscillatory activity in the theta band $(\sim 6 \mathrm{~Hz})$ has also been found in association with levodopa-induced dyskinesias and impulsivity induced by dopaminergic treatments (Alonso-Frech et al., 2006; Rodriguez-Oroz et al., 2011). Therefore, it is quite likely that pallidotomy eliminates dyskinesias by interrupting abnormal synchronization in BG output (Brown and Eusebio, 2008).

With regards to the second paradox, it is now recognized that animals with DA depletion and patients with PD demonstrate an impaired learning and execution of routines and habits and that PD patients with lesions of the BG motor output, after combined pallidotomy/subthalamotomy procedures, showed impaired acquisition of implicit learning (Obeso et al., 2009; Redgrave et al., 2010). Thus, lesions of the sensorimotor circuit of the BG are associated with deficits, but these are not apparent under normal conditions.

\section{THE PRESENT}

Several developments have occurred in the many years since the model was established, and these have had a corresponding impact on our understanding of the function(s) of the BG and their pathological derangement (Wichmann et al., 2011). Details are available in recent reviews and in the current volume of Frontiers in Neuroanatomy. Here, we summarize only the major areas of development. An updated version of the classical "box and arrows" model is provided in Figure 1.

\section{"DIRECT" AND "INDIRECT" PATHWAYS}

It is now recognized that the BG cannot be seen as a "go through" structure, whereby connectivity and functional interactions occur uni-directionally along cortico-BG-thalamo-cortical circuits (Graybiel, 2008; Obeso et al., 2008).

Revision of the original concept of the "indirect" pathway has been quite considerable in recent years. The STN is thus no longer placed in-between the GPe and GPi in the "indirect" circuit, but is now considered as another input station of the BG that receives afferents from the cerebral cortex (Inase et al., 1999; Nambu et al., 2000, 2002), thalamus (Lanciego et al., 2004) and brainstem (reviewed by Martínez-González et al., 2001, this issue). Moreover, besides the well-known STN output projections (i.e., GPe, $\mathrm{GPi} / \mathrm{SNr}$, and PPN nuclei), the presence of direct STN projections reaching the ventral thalamus has been often neglected and yet recently confirmed in the MPTP monkey model (Rico et al., 2010). Reciprocal connections between the GPe and the striatum (Sato et al., 2000) and between the striatum and DA neurons in the SNc (Haber et al., 2000) have been recognized. Finally, the STN-GPe-GPi form a microcircuit where GPe appears strategically placed to control BG output activity (Obeso et al., 2006). Accordingly, the exclusive feed-forward nature of information processing within the BG is not the only major feature of BG functional organization.

The concept of parallel cortico-BG loops has been largely confirmed in humans by functional magnetic resonance imaging ( $\mathrm{fMRI}$ ) and positron emission tomography (PET) studies and also by cortical magnetoencephalography and LFPs recorded from the STN. Activation studies have revealed a topographical segregation according to the requested task and underlying functions. Activation in the posterior (i.e., sensorimotor) putamen was consistently reported for any movement and presented a somatotopical organization, with the leg lying dorsal, face ventral and arm in-between, as expected. Preparatory activation as well as finger movement sequencing was located more rostrally in the anterior putamen, while activation of the associative territory was observed during tasks such as motor internal representation, and the selection and planning of sequential actions (Lehericy et al., 2005). 


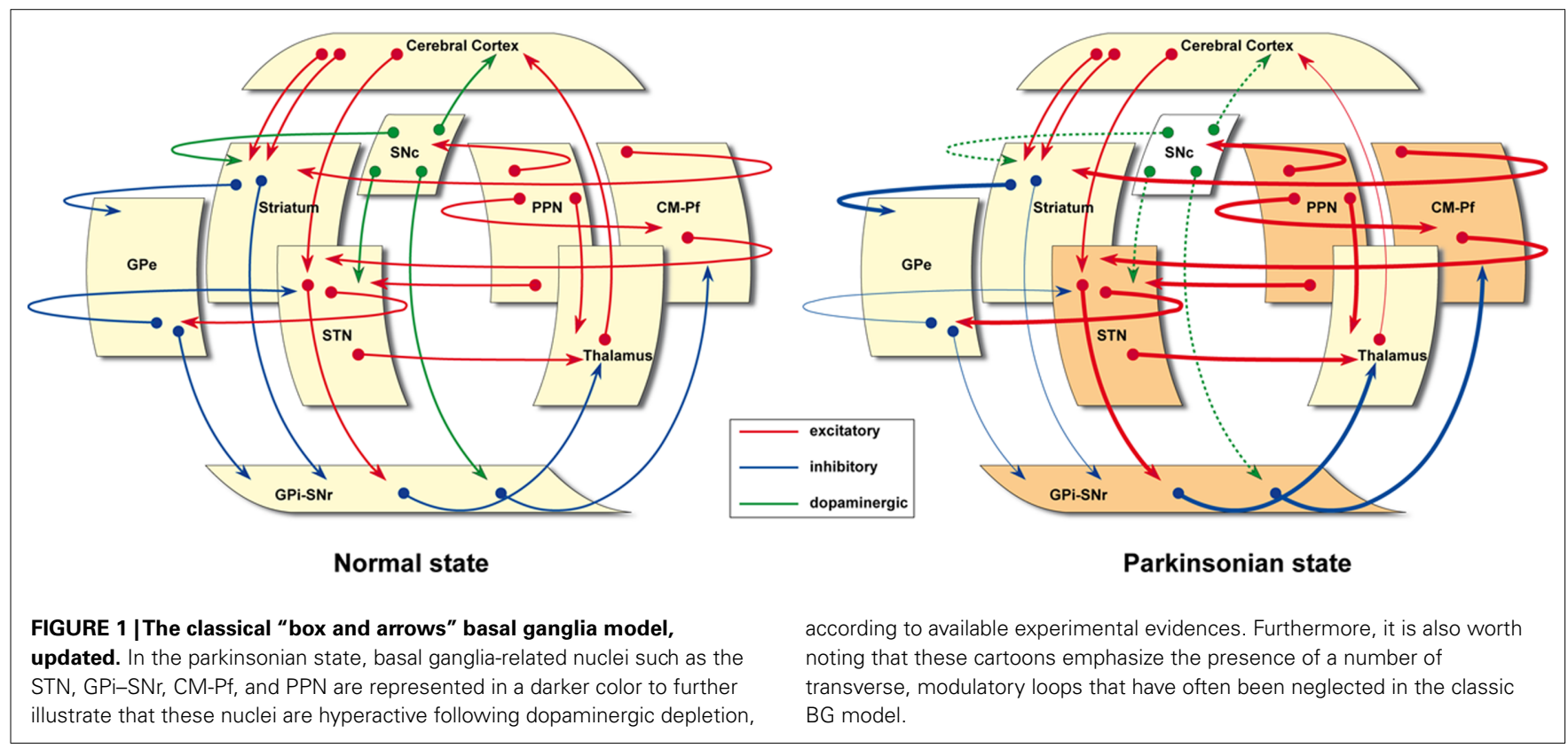

In addition to cortical input, the BG receive afferents from several sub-cortical nuclei (caudal intralaminar nuclei, PPN, superior colliculus, locus coeruleus, and raphe nuclei, among others) and, most prominently, from the thalamus (McHaffie et al., 2005).

\section{INTERNEURONS}

The original model did not take into account the role of striatal interneurons. BG nuclei are generally devoid of interneurons, the exception being the striatum which contains several types (reviewed in the accompanying manuscripts by Tepper et al., 2011 and Pisani et al., 2011, this issue). The cholinergic, tonically active neurons (TANs) and the GABAergic fast-spiking interneurons (FSIs) are the most prominent types of striatal interneurons. TANs modulate MSN excitability by pre-synaptic inhibition of cortical glutamatergic input, and mediate DAergic mechanisms leading to long-term depression (LTD). FSIs are less abundant but mediate feed-forward inhibition which together with axon collaterals from MSNs provide intrastriatal inhibition. These inhibitory effects seem particularly well suited to cause intense activation of some micro-regions of the striatum while inhibiting less active regions, i.e., they serve as a substrate for the selection process.

\section{THE DOPAMINERGIC SYSTEM}

The DAergic system projection innervates not only the striatum but also other BG structures such as the STN, GPe, and GPi, as well as the cortex, limbic system, and thalamus (Smith and Villalba, 2008). Data regarding how DA acts, particularly in the striatum, has changed considerably since the model was created. The nigro-striatal projection is now conceived as topographically subdivided into medial-ventral and dorso-lateral projections, which are highly divergent. DA secretion exerts a wide tonic -volume conduction- modulatory effect on striatal excitability and a more focal, SNc firing-related, phasic synaptic effect. It turns out that a majority of striatal DAergic synapses make "open" contacts with target neurons (Moss and Bolam, 2008), indicating the importance of tonic release. On the other hand, phasic changes in the dopaminergic response have been interpreted as an error signal that informs the corticostriatal system of the discrepancy between the prediction of a reward and its actual occurrence. This hypothesis sustains a major role for the DAergic system in reinforcement learning. However, it is now known that DA SNc neurons encode reward and non-reward events and are involved in other functions such as orientation, motivational driving and cognitive processing (Bromberg-Martin et al., 2010).

Another important issue under debate is represented by the D1-D2 dichotomy and its role in striatal output. DA is known to exert a dual effect on striatofugal neurons depending on the types of DA receptors present in the postsynaptic element. This reasoning represents one of the major foundations of the present BG model. Recently, anatomical, neurochemical and electrophysiological evidence (reviewed by Bertán-González et al., this issue) has supported the rather segregated distribution of D1 and D2 receptors within different types of striatofugal neurons. However, such a sharp degree of complementary expression for D1/D2 receptors in the two different populations of striatofugal MSNs still remains somewhat controversial and will probably require further elucidation. The recent introduction of BAC-transgenic mice permitted an estimation showing that only a small number of striatofugal MSNs (less than 6\% in the dorso-lateral striatum) coexpress D1 and D2 receptors (Bertran-Gonzalez et al., 2010). These newly available data challenge earlier results showing that roughly half of striatal MSNs co-express D1-like and D2-like receptors (as reviewed in Bertrán-González et al., this issue). It is also worth noting that anatomical data have their own limitations, including a limited number of neurons being reconstructed, the lack of information on the relative importance of each axon collateral compared to the main axonal terminal field, as well as the potential presence of tracer uptake by fibers of passage when dealing with retrograde tracing experiments. 
Besides nigro-striatal projections, in the past few years accumulating evidence showing that DA also exerts an important effect on BG nuclei other than the striatum have been made available (Smith and Villalba, 2008). These projections -named the nigro-extrastriatal pathway- are likely to sustain a key role in BG pathophysiology, particularly in compensatory mechanisms underlying earlier stages of PD. As reviewed by Rommelfanger and Wichmann in this issue, BG nuclei such as the GPe, GPi, STN, and $\mathrm{SNr}$ are innervated by nigral DAergic terminals. Both D1 and D2 receptors (and to a lesser extent D3, D4, and D5 receptors) are found in most of these nuclei. The role played by DA (excitation or inhibition) ultimately depends on the type of receptor activated as well as on the pre or postsynaptic localization of a given DA receptor. Recently, attention has been paid to the effects of DA on extra-striatal nuclei and the implications for compensatory mechanisms in PD (see Rommelfanger and Wichmann, this issue).

\section{STRIATAL AFFERENT SYSTEMS AND SUB-CORTICAL LOOPS}

The importance of sub-cortical interactions with the BG has been extensively documented in the last decade (McHaffie et al., 2005). Special attention has been dedicated during this time to define the relationship between cortical and thalamic glutamatergic afferents (Castle et al., 2005). In addition, the presence of bilateral corticostriatal projections must also be taken into consideration. Ipsilateral corticostriatal projections arise in the rat from two different populations of corticofugal neurons, known as intratelencephalic neurons (IT neurons) and pyramidal tract neurons (PT type), whereas contralateral afferents originate only from PT type neurons. Importantly, whereas the inputs of IT type neurons mainly reach striatal MSNs giving rise to the "direct" pathway, PT neurons preferentially target MSNs projecting through the indirect pathway (reviewed in Reiner et al., 2010, this issue). Furthermore, the thalamostriatal system also represents a major source of ipsilateral glutamatergic projections to the striatum, an afferent source that has often been neglected.

\section{PATHOPHYSIOLOGY}

\section{Animal models}

The major functional sub-divisions of the BG have been further delineated by behavioral studies in monkeys. Manipulations of different functional territories of the BG nuclei are associated with emotional, learning, and motor manifestations. Specifically, focal injections of bicucculline or muscimol to block specific sub-regions of the striatum, GPe and STN, produced dyskinesias, stereotopies, and hyperactivity among other behavioral abnormalities when injected into the the postero-lateral (motor) segment, associative, and limbic regions (Karachi et al., 2009). Injections in the ventral striatum also elicited sexual responses (erection and ejaculation) and vomiting (Karachi et al., 2009). These studies indicate that interruption of different $\mathrm{BG}$ sub-regions gives rise to abnormal movements, behaviors, and mood changes.

Two recent studies in the rat applying optogenetics have provided strong support to the classic model. Kravitz et al. (2010) showed that selective stimulation of MSNs expressing D2 receptors ("indirect" circuit) provoked movement arrest, while activation of MSNs expressing D1 receptors led to movement activation. This essentially confirmed the notion that the "indirect" and "direct" circuits are in functional equilibrium, respectively inhibiting and facilitating movement. Furthermore, blockade of DARPP-32 to inhibit D1-expressing MSNs abolish levodopa-induced dyskinesias (Bateup et al., 2010), thus providing clear support to the key role of the "direct" circuit in mediating this complication, and well in keeping with several previous studies (Aubert et al., 2005).

\section{Clinical studies}

A number of major advances have occurred by recording LFPs via electrodes implanted in the STN (or GPi) for DBS. In the parkinsonian state there is an increment in the power at around $11-30 \mathrm{~Hz}$, which is drastically reduced after taking levodopa and occurs concurrently with motor improvement (Brown, 2003; Gatev et al., 2006). Time-locked changes in STN activity have also been recorded with emotional stimuli such as the showing of emotionally laden and neutral pictures, during behavioral tasks requiring decisions between relevant or non-relevant stimuli for a given task, and during movement observation (Sauleau et al., 2009; Alegre et al., 2010). Many of these studies have been performed in the "off" (parkinsonian) medication state. In the "on" medication state when, presumably, DA deficits have been restored to some extent, the major changes consist of a marked reduction in beta power activity and enhancement of gamma activity (Brown, 2003) together with, in patients with levodopa-induced dyskinesias, a significant increment in slow $(6-8 \mathrm{~Hz})$ oscillations mainly from the dorsal STN (Brown, 2003).

Thus, the more recent neurophysiological data may be taken in support for activation of the BG both before and after movement onset and during cognitive and emotionally related tasks. Moreover, the motor vs. limbic, dorsal vs. ventral distribution appears substantiated, at least for the STN, as does the differential involvement of discrete circuits in abnormal movements and behaviors.

A new concept resides in the role of the BG in inhibition (Eagle and Baunez, 2010). This is applied to activities such as stopping an already ongoing order to move, halting an already initiated movement and suppressing an inconvenient behavior or desire. The STN has been particularly implicated in these inhibitory functions, which are essential for adequate performance of daily life activities (Aron and Poldrack, 2006).This function has recently been shown to be abnormal in PD patients and will be further delineated in upcoming studies.

\section{The origin of Parkinson's disease}

The real challenge at present and indeed in the future is for evidence to be uncovered to provide definitive understanding of the factors associated with the onset of the neurodegeneration process. Herein, a major effort is currently underway and will continue in the near future, to delineate the features explaining the vulnerability of dopaminergic neurons in the SNc. Although the main underlying causes sustaining this cellular degeneration remain to be fully elucidated, a number of factors have been identified, including those related to mitochondrial dysfunction, impairment of proteostasis, neuroinflammatory phenomena, glutamate-driven excitotoxicity, and even normal aging. Moreover, instead of there being a single mechanism, it is more likely that a combination of 
different factors ultimately leads to the loss of DAergic neurons (Obeso et al., 2010). It is also likely that functional connectivity and physiological features will play an important role in determining selective vulnerability of ventro-lateral SNpc neurons in PD. This could become another relevant contribution of functional anatomy to improving our understanding and treatment of PD.

\section{THE FUTURE}

The model of the BG has paved the way toward many new developments and ignited renewed interest in the application of functional neurosurgery, which by itself may be considered a significant contribution to translational neuroscience. However, the imperfections and inaccuracies of the model are not minor and indeed, were noticed early on in the development of these concepts.

Nambu (2008) outlined in a lucid essay the seven main problems of the BG model. While major problems have been, on the whole, addressed, some of these issues have now been overshadowed by a wealth of data and information. For instance, we can now take for granted that the classic concept of "direct" and "indirect" pathways is no longer tenable, particularly because of the plethora of connections identified between the GPe and the STN with other nuclei. Similarly, the limitations of the "rate model" to explain BG pathophysiology are well accepted. However, many issues and queries remain open to study and interpretations. Among these, the following may be highlighted: (1) Convergence. There has to be a way whereby neuronal activity in the different loops, and even within the motor circuit, converge, so that a final coherent signal is provided for facilitating a given movement or behavior. (2) Nigro-striatal and nigro-extrastriatal projections. Much work needs to be done to recognize the putative topography and features of neuronal activity in register with ascending projections to the striatum and other nuclei. This is seen as particularly important regarding $\mathrm{PD}$, where the most vulnerable neurons

\section{REFERENCES}

Albin, R. L., Young, A. B., and Penney, J. B. (1989). The functional anatomy of basal ganglia disorders. Trends Neurosci. 12, 366-375.

Alegre, M., Rodríguez-Oroz, M. C., Valencia, M., Pérez-Alcázar, M., Guridi, J., Iriarte, J., Obeso, J. A., and Artieda, J. (2010). Changes in subthalamic activity during movement observation in Parkinson's disease: is the mirror system mirrored in the basal ganglia? Clin. Neurophysiol. 121, 414-425.

Alexander, G. E., DeLong, M. R., and Strick, P. L. (1986). Parallel organization of functionally segregated circuits linking basal ganglia and cortex. Annu. Rev. Neurosci. 9, 357-381.

Alonso-Frech, F., Zamarbide, I., Alegre, M., Rodríguez-Oroz, M. C., Guridi, J., Manrique, M., Valencia, M., Artieda, J., and Obeso, J. A. (2006). Slow oscillatory activity and levodopa-induced dyskinesias in Parkinson's disease. Brain 129, 1748-1757.

Aron, A. R., and Poldrack, R. A. (2006). Cortical and subcortical contributions to stop signal response inhibition: role of the subthalamic nucleus. J. Neurosci. 26, 2424-2433.

Aubert, I., Guigoni, C., Håkansson, K., Li, Q., Dovero, S., Barthe, N., Bioulac, B. H., Gross, C. E., Fisone, G., Bloch, B., and Bezard, E. (2005). Increased D1 dopamine receptor signaling in levodopa-induced dyskinesia. Ann. Neurol. 57, 17-23.

Aziz, T. Z., Peggs, D., Sambrook, M. A., and Crossman, A. R. (1991). Lesion of the subthalamic nucleus for the alleviation of 1-methyl-4phenyl-1,2,3,6-tetrahydropyridine (MPTP)-induced parkinsonism in the primate. Mov. Disord. 6, 288-292.

Bateup, H. S., Santini, E., Shen, W., Birnbaum, S., Valjent, E., Surmeier, D. J., Fisone, G., Nestler, E. J., and Greengard, P. (2010).

are the ones projecting to the posterior putamen. Moreover, the temporal evolution and functional implications of putative DA depletion in the nigro-extrastriatal connections awaits further clarification. (3) Bilateral representation. Up to now, most studies of the BG, and certainly the Model, contemplated connections and functions in a rather unilateral fashion. However, most movements and behaviors involve bilateral and synchronous activity. It is likely, therefore, that neuronal activity in the BG is under bilateral and reciprocal influences. (4) Basic Functional Support. Substantial evidence has been provided to demonstrate that inhibition or pausing of neuronal activity in the output of the BG is the primary mechanism for facilitation, while increased neuronal firing possibly sustains inhibition or arrest of actions. Whether or not the same basic principle sustains activity in the associative and limbic domains is not known and should be clarified in the near future.

In sum, a wide spectrum of future research can be envisaged, and important advances are already taking place. For instance, the ability to dissect out and study the function of specific circuits by using advanced morphological techniques and the application of optogenetics will provide even greater insight into the functional organization of the BG. Studies in awake animals, particularly in monkeys, will continue to illustrate crucial aspects of neuronal activity in relation to specific tasks. Importantly, human studies are now possible thanks to the development of powerful and sophisticated neuroimaging and neurophysiological techniques, which provide far better resolution of BG activation and distortions under normal and pathological conditions. The function(s) of the BG should no longer be considered "as dark as the basement of my house" as ironically indicated by the British neurologist Kinnier Wilson at the beginning of the twentieth century (Wilson, 1925). Nevertheless, astute experimental designs and sharp clinical observations will remain foremost priorities to further enlighten our understanding of the BG.

Distinct subclasses of medium spiny neurons differentially regulate striatal motor behaviors. Proc. Natl. Acad. Sci. U.S.A. 107, 14845-14850.

Bertran-Gonzalez, J, Hervé, D., Girault, J.-A., and Valjent, E. (2010). What is the degree of segregation between striatonigral and striatopallidal projections? Front. Neuroanat. 4:136. doi: 10.3389/fnana.2010. 00136

Bromberg-Martin, E. S., Matsumoto, M., and Hikosaka, O. (2010). Dopamine in motivational control: rewarding, aversive, and alerting. Neuron 68, 815-834.

Brown, P. (2003). Oscillatory nature of human basal ganglia activity: relationship to the pathophysiology of Parkinson's disease. Mov. Disord. 18, 357-363.

Brown, P., and Eusebio, A. (2008). Paradoxes of functional neurosurgery: clues from basal ganglia recordings. Mov. Disord. 23, 12-20.
Brown, P., Oliviero, A., Mazzone, P., Insola, A., Tonali, P., and Di Lazzaro, V. (2001). Dopamine dependency of oscillations between subthalamic nucleus and pallidum in Parkinson's disease. J. Neurosci. 21, 1033-1038.

Castle, M., Aymerich, M. S., SánchezEscobar, C., Gonzalo, N., Obeso, J. A., and Lanciego, J. L. (2005). Thalamic innervation of the direct and indirect basal ganglia circuits in the rat: Ipsi- and contralateral projections. J. Comp. Neurol. 483, 143-153.

Chevalier, G., and Deniau, J. M. (1990). Disinhibition as a basic process in the expression of striatal functions. Trends Neurosci. 13, 277-281.

Crossman, A. R. (1990). A hypothesis on the pathophysiological mechanisms that underlie levodopa- or dopamine agonist-induced dyskinesia in Parkinson's disease: implications for future strategies in treatment. Mov. Disord. 5, 100-108. 
DeLong, M. R. (1990). Primate models of movement disorders of basal ganglia origin. Trends Neurosci. 13, 281-285.

Eagle, D. M., and Baunez, C. (2010). Is there an inhibitory-responsecontrol system in the rat? Evidence from anatomical and pharmacological studies of behavioral inhibition. Neurosci. Biobehav. Rev. 34, 50-72.

Gatev, P., Darbin, O., and Wichmann, T. (2006). Oscillations in the basal ganglia under normal conditions and in movement disorders. Mov. Disord. 21, 1566-1577.

Gerfen, C. R., Engber, T. M., Mahan, L. C., Susel, Z., Chase, T. N., Monsma, F. J. Jr., and Sibley, D. R. (1990). D1 and D2 dopamine receptor regulated gene expression of striatonigral and striatopallidal neurons. Science 250, 1429-1431.

Graybiel, A. M. (2008). Habits, rituals, and the evaluative brain. Annu. Rev. Neurosci. 31, 359-387.

Guridi, J., Herrero, M. T., Luquin, M. R., Guillén, J., Ruberg, M., Laguna, J., Vila, M., Javoy-Agid, F., Agid, Y., Hirsch, E., and Obeso, J. A. (1996). Subthalamotomy in parkinsonian monkeys. Behavioural and biochemical analysis. Brain 119, 1717-1727.

Haber, S. N., Fudge, J. L., and McFarland, N. R. (2000). Striatonigrostriatal circuits in primates form an ascending spiral from the shell to the dorsolateral striatum. J. Neurosci. 20, 2369-2382.

Inase, M., Tokuno, H., Nambu, A., Akazawa, T., and Takada, M. (1999). Corticostriatal and corticosubthalamic input zones from the presupplementary motor area in the macaque monkey: comparison with the input zones from the supplementary motor area. Brain Res. 833, 191-201.

Karachi, C., Grabli, D., Baup, N., Mounayar, S., Tandé, D., François, C., and Hirsch, E. C. (2009). Dysfunction of the subthalamic nucleus induced behavioral and movement disorders in monkeys. Mov. Disord. 24, 1183-1192.

Kravitz, A. V., Freeze, B. S., Parker, P. R., Kay, K., Thwin, M. T., Deisseroth, K., and Kreitzer, A. C. (2010). Regulation of parkinsonian motor behaviours by optogenetic control of basal ganglia circuitry. Nature 466, 622-626.

Lanciego, J. L., Gonzalo, N., Castle, M. Sanchez-Escobar, C., Aymerich, M S., and Obeso, J. A. (2004). Thalamic innervation of striatal and subthalamic neurons projecting to the rat entopeduncular nucleus. Eur. J. Neurosci. 19, 1267-1277.

Lehericy, S., Benali, H., Van de Moortele, P. F., Pélégrini-Issac, M., Waechter T., Ugurbil, K., and Doyon, J. (2005). Distinct basal ganglia territories are engaged in early and advanced motor sequence learning. Proc. Natl. Acad. Sci. U.S.A. 102, 12566-12571.

Marsden, C. D., and Obeso, J. A. (1994). The functions of the basal ganglia and the paradox of stereotaxic surgery in Parkinson's disease. Brain 117, 877-897.

McHaffie, J. G., Stanford, T. R., Stein, B. E., Coizet, V., and Redgrave, P. (2005). Subcortical loops through the basal ganglia. Trends Neurosci. 28, 401-407.

Moss, J., and Bolam, J. P. (2008). A dopaminergic axon lattice in the striatum and its relationship with cortical and thalamic terminals. $J$. Neurosci. 28, 11221-11230.

Nambu, A. (2008). Seven problems on the basal ganglia. Curr. Opin. Neurobiol. 18, 595-604.

Nambu, A., Tokuno, H., Hamada, I., Kita, H., Imanishi, M., Akazawa, T. Ikeuchi, Y., and Hasegawa, N. (2000). Excitatory cortical inputs to pallidal neurons via the subthalamic nucleus in the monkey. J. Neurophysiol. 84 289-300.

Nambu, A., Tokuno, H., and Takada, M. (2002). Functional significance of the cortico-subthalamo-pallidal 'hyperdirect' circuit. Neurosci. Res. 43, 111-117.

Obeso, J. A., Jahanshahi, M., Alvarez, L., Macias, R., Pedroso, I., Wilkinson, L., Pavon, N., Day, B., Pinto, S., Rodríguez-Oroz, M. C., Tejeiro, J., Artieda, J., Talelli, P., Swayne, O., Rodríguez, R., Bhatia, K., RodriguezDiaz, M., Lopez, G., Guridi, J., and Rothwell, J. C. (2009). What can man do without basal ganglia motor output? The effect of combined unilateral subthalamotomy and pallidotomy in a patient with
Parkinson's disease. Exp. Neurol. 220 283-292.

Obeso, J. A., Rodriguez, M. C., and DeLong, M. R. (1997). Basal ganglia pathophysiology. A critical review. Adv. Neurol. 74, 3-18.

Obeso, J. A., Rodríguez-Oroz, M. C. Benitez-Temino, B., Blesa, F. J. Guridi, J., Marin, C., and Rodriguez, M. (2008). Functional organization of the basal ganglia: therapeutic implications for Parkinson's disease. Mov. Disord. 23(Suppl. 3), S548-S559.

Obeso, J. A., Rodriguez-Oroz, M. C., Goetz, C. G., Marin, C., Kordower, J. H., Rodriguez, M., Hirsch, E. C., Farrer, M., Schapira, A. H., and Halliday, G. (2010). Missing pieces in the Parkinson's disease puzzle. Nat. Med. 16, 653-661.

Obeso, J. A., Rodriguez-Oroz, M. C. Javier Blesa, F., and Guridi, J. (2006). The globus pallidus pars externa and Parkinson's disease. Ready for prime time? Exp. Neurol. 202, $1-7$.

Penney, J. B. Jr., and Young, A. B. (1986) Striatal inhomogeneities and basal ganglia function. Mov. Disord. 1, 3-15.

Redgrave, P., Rodriguez, M., Smith, Y., Rodriguez-Oroz, M. C., Lehericy, S., Bergman, H., Agid, Y., DeLong, M. R., and Obeso, J. A. (2010). Goal-directed and habitual control in the basal ganglia: implications for Parkinson's Disease. Nat. Rev. Neurosci. 11 , 760-772.

Rico, A. J., Barroso-Chinea, P., ContePerales, L., Roda, E., GómezBautista, V., Gendive, M., Obeso, J. A., and Lanciego, J. L. (2010). A direct projection from the subthalamic nucleus to the ventral thalamus in monkeys. Neurobiol. Dis. 39, 381-392.

Rodriguez-Oroz, M. C., LopezAzcarate, J., Garcia-Garcia, D. Alegre, M., Toledo, J., Valencia, M., Guridi, J., Artieda, J., and Obeso, J. A. (2011). Involvement of the subthalamic nucleus in impulse control disorders associated with Parkinson's disease. Brain 134, 36-49.

Sato, F., Lavallée, P., Lévesque, M. and Parent, A. (2000). Single-axon tracing study of neurons of the external segment of the globus pallidus in primate. J. Comp. Neurol. 417, 17-31.

Sauleau, P., Eusebio, A., Thevathasan, W., Yarrow, K., Pogosyan, A., Zrinzo, L., Ashkan, K., Aziz, T., Vandenberghe, W., Nuttin, B., and Brown, P. (2009). Involvement of the subthalamic nucleus in engagement with behaviourally relevant stimuli. Eur. J. Neurosci. 29, 931-942.

Smith, Y., and Villalba, R. (2008). Striatal and extrastriatal dopamine in the basal ganglia: an overview of its anatomical organization in normal and Parkinsonian brains. Mov. Disord. 23(Suppl. 3), S534-S547.

Wichmann, T., Bergman, H., and DeLong, M. R. (1994). The primate subthalamic nucleus. III. Changes in motor behavior and neuronal activity in the internal pallidum induced by subthalamic inactivation in the MPTP model of parkinsonism. J. Neurophysiol. 72, 521-530.

Wichmann T., DeLong, M. R., Guridi J., and Obeso, J. A. (2011). Milestones in research on the pathophysiology of Parkinson's disease. Mov. Disord. 26, 1032-1041.

Wilson, S. A. K. (1925). Disorders of motility and tone. Lancet I, 1-103.

Conflict of Interest Statement: The authors declare that the research was conducted in the absence of any commercial or financial relationships that could be construed as a potential conflict of interest.

Received: 25 May 2011; accepted: 29 June 2011; published online: 12 July 2011.

Citation: Obeso JA and Lanciego JL (2011) Past, present, and future of the pathophysiological model of the basal ganglia. Front. Neuroanat. 5:39. doi: 10.3389/fnana.2011.00039

Copyright (C) 2011 Obeso and Lanciego. This is an open-access article subject to a non-exclusive license between the authors and Frontiers Media SA, which permits use, distribution and reproduction in other forums, provided the original authors and source are credited and other Frontiers conditions are complied with. 